\title{
NORMAL SURFACES IN NON-COMPACT 3-MANIFOLDS
}

\author{
ENSIL KANG \\ (Received 30 September 2002; revised 7 November 2003) \\ Communicated by C. D. Hodgson
}

\begin{abstract}
We extend the normal surface $Q$-theory to non-compact 3-manifolds with respect to ideal triangulations. An ideal triangulation of a 3-manifold often has a small number of tetrahedra resulting in a system of $Q$ matching equations with a small number of variables. A unique feature of our approach is that a compact surface $F$ with boundary properly embedded in a non-compact 3-manifold $M$ with an ideal triangulation with torus cusps can be represented by a normal surface in $M$ as follows. A half-open annulus made up of an infinite number of triangular disks is attached to each boundary component of $F$. The resulting surface $\hat{F}$, when normalized, will contain only a finite number of $Q$-disks and thus correspond to an admissible solution to the system of $Q$-matching equations. The correspondence is bijective.
\end{abstract}

2000 Mathematics subject classification: primary 57M99; secondary 57M10.

Keywords and phrases: 3-manifold, normal surface, knot.

\section{Introduction}

In this paper we develop a normal surface theory that provides an efficient way to represent spanning surfaces of knots using ideal triangulations of the knot complements. A normal surface is a properly embedded surface in a compact, triangulated 3-manifold which intersects each tetrahedron in elementary disks. There are three quadrilateral and four triangular disk types. There is a one-to-one correspondence between normal surfaces in a 3-manifold with a fixed triangulation and admissible solutions to a system of linear equations of $7 t$ variables, where $t$ is the number of tetrahedra of the triangulation and each variable represents the number of disks of each elementary disk type. This algebraic representation of normal surfaces was introduced by Haken [3].

This study was supported by research funds from Chosun University 2004 .

(c) 2005 Australian Mathematical Society 1446-7887/05 \$A2.00+0.00 
In [11], Tollefson observed that a normal surface without trivial components is completely determined by its quadrilateral disks alone. Thus $4 t$ variables are not needed and only the $3 t$ variables associated with the $Q$-disks need be involved. There are also fewer $Q$-matching equations than in the original approach. In this paper we extend this approach to apply to ideal triangulations which often have a small number of tetrahedra.

Let $M$ be a non-compact 3-manifold with a finite ideal triangulation with torus cusps. We represent compact surfaces with boundary in $\mathbb{R}^{n}$ by their interiors properly embedded in $M$ as normal surfaces containing only a finite number of quadrilateral disks and infinite number of triangular disks. A regular neighbourhood of a boundary component of such a surface is represented by an infinite annulus consisting of only triangular disks. In an ideal triangulation of a non-compact 3-manifold $M$, regular normal surface theory only works for closed surfaces. Bounded surfaces such as Seifert surfaces can only be represented by using an infinite number of triangles. Normal surface $Q$-theory only deals with quadrilateral disks to characterize a normal surface by a finite-tuple of integers. We use the $Q$-theory to study normal surfaces representing both closed and bounded surfaces in a knot complement with an ideal triangulation.

An ideal triangulation of a non-compact 3-manifold often has a small number of tetrahedra resulting in an algebraic representation of normal surfaces with a small number of disk types. This leads to a system of $Q$-matching equations with a small number of variables each of which represents the number of disks of a $Q$-disk type. Admissible solutions to the system of $Q$-matching equations correspond bijectively to normal surfaces in the 3-manifold. Although we give an algorithm to construct a normal surface corresponding to a given admissible $Q$-solution, one can determine the surface modulo 'trivial normal surfaces' from the quadrilateral disks alone without attaching triangular disks.

\section{Normal surface $Q$-theory for ideal triangulations}

Let $M$ be a non-compact 3-manifold with an ideal triangulation $\Im$ with torus cusps. Let $\tau$ be an ideal tetrahedron of $\Im$. We can view $\tau$ as the quotient of an abstract tetrahedron $\tilde{\tau}$ with no self-intersections under a quotient map $q_{\tau}: \hat{\tau} \rightarrow \tau$ and with its vertices removed. We define an elementary disk in $\tau$ to be the quotient of an elementary disk in $\hat{\tau}$ under the map $q_{\mathrm{r}}$. Thus we allow identifications along boundaries of elementary disks in $\tau$. We have exactly seven elementary disk types in each tetrahedron of $\mathfrak{J}$, four triangular disk ( $T$-disk) types and three quadrilateral disk ( $Q$-disk) types, ignoring possible identification along boundaries. Throughout this paper, we only deal with 3-manifolds decomposed into a finite number of ideal 
tetrahedra. We often omit the term 'ideal' to indicate cells in ideal triangulations. A normal surface $F$ is a properly embedded surface in $M$ which intersects each tetrahedron only in elementary disk types. A properly embedded surface in a 3manifold $M$ is defined as a surface which is the image of an embedding $f: F \rightarrow M$ such that $f^{-1}(\partial M)=\partial F$ and preimages of compact sets are compact. The interior of any properly embedded surface in a compact 3-manifold is properly embedded in the interior of the 3-manifold. We say that $F$ represents a compact surface $G$ in $\mathbb{R}^{n}$ if there is a proper embedding $f: \stackrel{\circ}{G} \rightarrow M$ such that $f(\stackrel{\circ}{G})=F$. Let $F$ be a normal surface representing a compact surface $G$ with boundary. If we look at the behavior of $F$ in a small regular neighbourhood $U$ of an ideal vertex of $\Im, A=F \cap \bar{U}$ is a half-open infinite annulus consisting of only $T$-disks and possibly some portions of $T$-disks along $\partial A$ (see Figure 1). For convenience, we call such an annulus an infinite trivial normal annulus.

For a compact 3-manifold with a triangulation $\Im$, the weight of a normal surface $F$, defined to be the number of points in the intersection of $F$ with the 1-skeleton of the triangulation, is given as a useful measure of $F$. But for a non-compact 3-manifold with an ideal triangulation, it does not make sense measuring a normal surface $F$ representing a surface with boundary with the weight. So we define a new system of measuring $F$, called the $Q$-weight and denoted by $w t_{Q} F$, to be the number of $Q$-disks of $F$. Since a geometric sum is only performed between normal surfaces with compatible quadrilaterals, the $Q$-weight is additive.
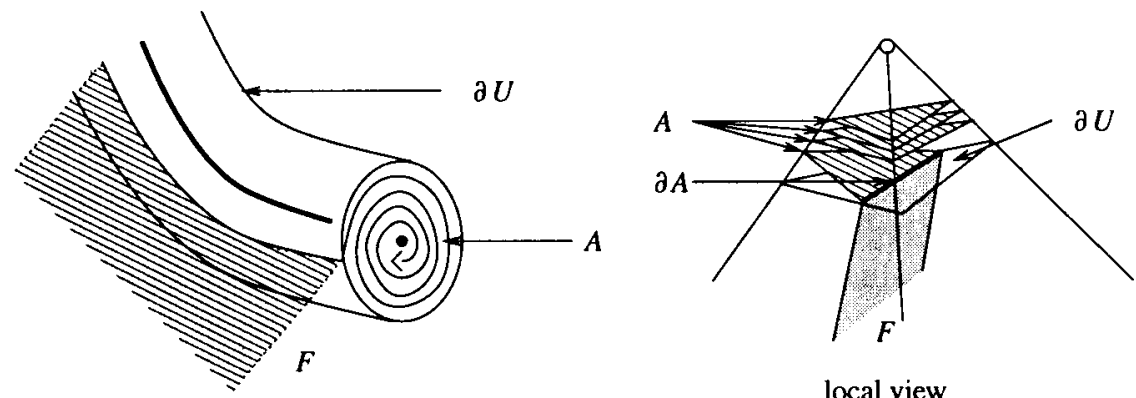

local view

FIGURE 1. A representation of a compact surface with boundary

Fix an ordering $d_{1}^{Q}, d_{2}^{Q}, \ldots, d_{3 t}^{Q}$ of all $Q$-disk types in $\Im$. There is a unique $3 t$-tuple representing the normal $Q$-coordinate $\vec{F}_{Q}=\left(x_{1}, x_{2}, \ldots, x_{3}\right)$ of $F$, where $x_{i}$ denotes the number of $Q$-disks of $d_{i}^{Q}$-type, $1 \leq i \leq 3 t$. Since $F$ is properly embedded, it follows that each component of a normal $Q$-coordinate is a non-negative integer. In the next section it will be shown that $F$ is uniquely determined, up to trivial components, by the normal $Q$-coordinate $\vec{F}_{Q}$. A trivial component is a normal surface containing only triangular disks. A closed trivial component in $M$ is a link of an ideal vertex 


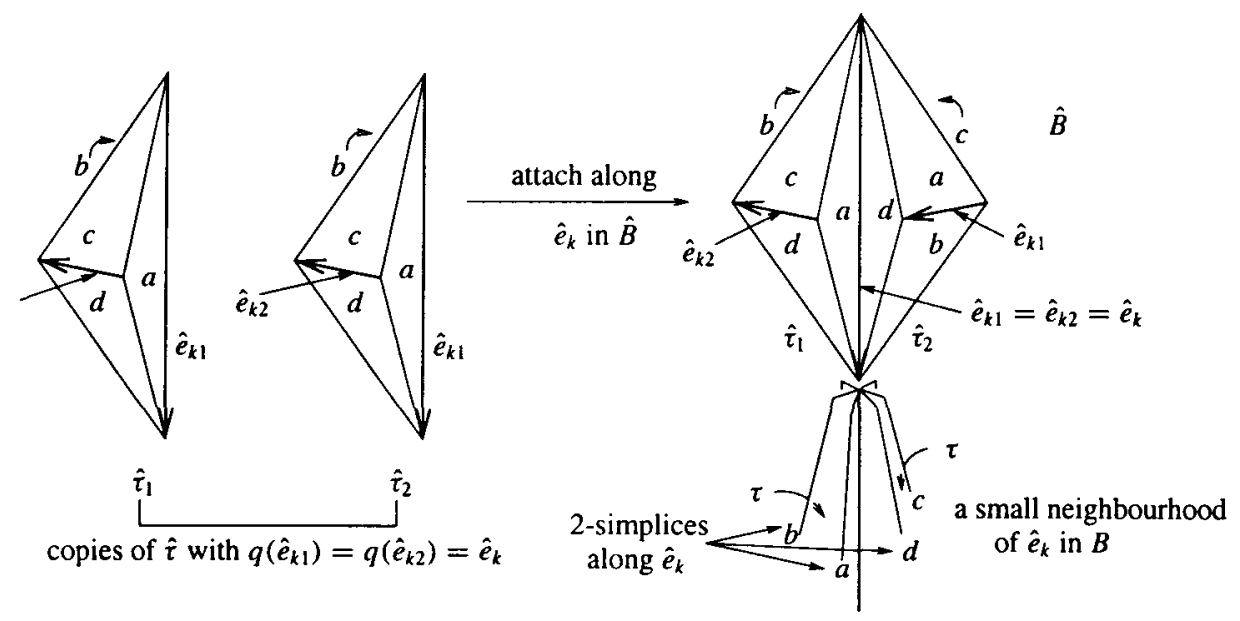

FIGURE 2. Gluing pattern to form an abstract polyhedron

which is a torus.

For a given $3 t$-tuple $\left(x_{1}, x_{2}, \ldots, x_{3 t}\right)$, there are two constraints to be satisfied for the tuple to be a normal $Q$-coordinate of a normal surface. The first constraint is that there is at most one $x_{i}$ from a tetrahedron which is non-zero. The second constraint is that it must satisfy a linear system of equations which represents the matching criterion of elementary disks along 1-simplices. The structure of a normal surface around a 1-simplex is completely determined by how the elementary disks along the 1 -simplex are glued together in the abstract polyhedron associated with the 1-simplex. Let $M^{\prime}$ be the compact 3-manifold obtained by removing the interiors of fixed regular neighbourhoods of ideal vertices of $\mathfrak{I}$. The boundary components of $M^{\prime}$ are trivial normal tori. We denote a 1 -simplex $e_{k}$ of $\Im$ by $\langle a b\rangle$, where $e_{k} \cap \partial M^{\prime}=\{a, b\}$, and call $a$ and $b$ the boundary vertices of $e_{k}$. Let $B_{k}$ be the union of all tetrahedra of $\Im$ containing $e_{k}=\langle a b\rangle$ as a face. Let $\tau$ be a tetrahedron in $B_{k}$ which is the quotient of an abstract tetrahedron $\hat{\tau}$ under the quotient map $q_{\tau}: \hat{\tau} \rightarrow \tau$. If $q_{\tau}^{-1}\left(e_{k}\right)$ consists of $n$ components $(n \leq 6)$, then take $n$ copies of $\hat{\tau}$, each associated with a component $\hat{e}_{k_{i}}$ of $q_{\tau}^{-1}\left(e_{k}\right)$. Repeat this for all tetrahedra in $B_{k}$. From this collection of abstract tetrahedra, form the abstract polyhedron $\hat{B}_{k}$ associated with the edge $e_{k}$ by gluing pairwise the faces of these tetrahedra around an edge $\hat{e}_{k}$ in such a way that a natural quotient map $q: \hat{B}_{k} \rightarrow B_{k}$ with $q\left(\hat{e}_{k}\right)=e_{k}$ and $\left.q\right|_{\hat{\tau}}=q_{\mathrm{\tau}}$ is well-defined (see Figure 2). Denote $\hat{e}_{k}=\langle\hat{a} \hat{b}\rangle$, where $\hat{a}$ and $\hat{b}$ are points in $\hat{e}_{k}$ such that $q_{\tau}(\hat{a})=a$ and $q_{\tau}(\hat{b})=b$. If an elementary $T$-disk $E$ in $\hat{B}_{k}$ is normal isotopic to a $T$-disk $\langle\hat{a} * *\rangle$ such that $q_{\mathrm{r}}(\langle\hat{a} * *\rangle)$ is a $T$-disk in a trivial normal torus of $\partial M^{\prime}$, we call $E$ an $a$-type $T$-disk. We define a b-type $T$-disk in the same manner. We call a spanning arc $\alpha$ in a 2-simplex of $\hat{\tau}$ an a-type arc (respectively b-type arc) if $\alpha$ is an edge of an $a$-type 
$T$-disk (respectively $b$-type $T$-disk) in the tetrahedron $\hat{\tau}$. For a given normal surface $F$ intersecting the edge $e_{k}$, a small neighbourhood $U_{k}$ of $e_{k}$ in $B_{k}$ intersects $F$ in a collection of pairwise disjoint disks meeting $e_{k}$. Since the quotient map $q: \hat{B}_{k} \rightarrow B_{k}$ maps a small neighbourhood of $\hat{e}_{k}$ homeomorpically onto a small neighbourhood of $e_{k}$, the union $\hat{D}$ of components of $q^{-1}\left(F \cap B_{k}\right)$ which intersect $\hat{e}_{k}$ is a disjoint union of disks which consist of elementary disks in $\hat{B}_{k}$ and $q(\hat{D}) \cap U_{k}=F \cap U_{k}$. Hence the gluing method of elementary disks in $\hat{D}$ along $\hat{e}_{k}$ dictates how the elementary disks of $F$ in $B_{k}$ are glued together along $e_{k}$. The matching criterion of elementary disks in an abstract polyhedron is described in the $Q$-matching equations in [11]. It is based on the observation that for a fixed 1-simplex, the number of $Q$-disks of positive sense is the same as the number of $Q$-disks of negative sense.

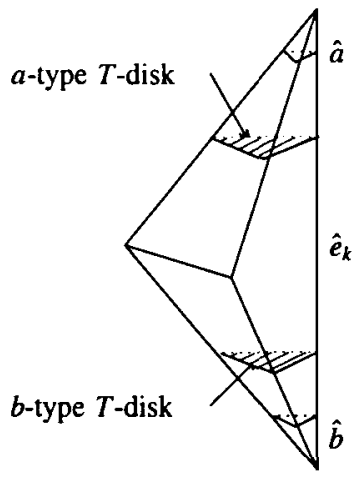

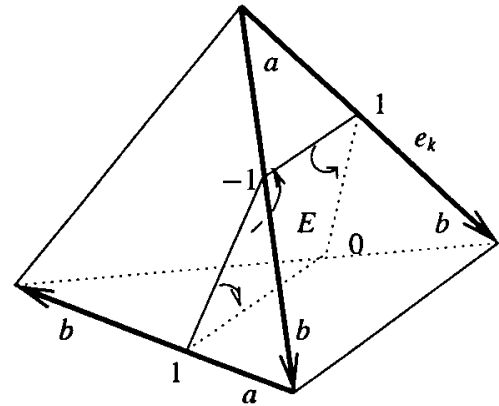

(The sense of $E$ associated with $e_{k}$ ) $=1$

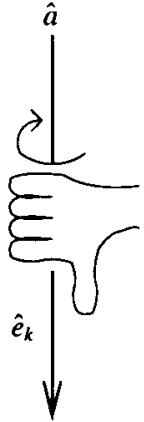

$\hat{b}$

FIGURE 3. The signs of corners of a $Q$-disk $E$ around an edge $e_{k}$

We here define the sense of a $Q$-disk associated with a fixed 1-simplex. Let $E$ be a $Q$-disk in a tetrahedron $\tau$ and $e_{k}$ a 1 -simplex with the positive orientation from $a$ to $b$ so that the positive orientation of $\hat{e}_{k}$ is from $\hat{a}$ to $\hat{b}$. We define the positive rotation around $\hat{e}_{k}$, by the right hand rule as shown in Figure 3. Let $v_{i}$ be a corner of $E$ on the edge $e_{k}$ and $\hat{E}_{i}$ the $Q$-disk in the abstract tetrahedron $\hat{\tau}_{i}$ in $\hat{B}_{k}$ such that $q_{\tau}\left(\hat{E}_{i}\right)=E$ and $q_{\tau}\left(\hat{v}_{i}\right)=v_{i}$, where $\hat{v}_{i}$ is the corner of $\hat{E}_{i}$ on $\hat{e}_{k}$. Define the sign $\delta_{k, i}$ of the corner $v_{i}$, relative to the edge $e_{k}$, to be +1 if the positive rotation around $\hat{e}_{k}$ carries the face of $\hat{\tau}_{i}$ containing the $a$-type arc of $\hat{E}_{i}$ through $\hat{\tau}_{i}$ to the face of $\hat{\tau}_{i}$ containing the $b$-type arc of $\hat{E}_{i}$ (see Figure 3). We say that the $Q$-disk $E$ is of the direction from a to $b$ around the corner, relative to $e_{k}=\langle a b\rangle$. The sign $\delta_{k, i}$ of $v_{i}$ is -1 if $E$ is of the direction from $b$ to $a$ around the corner. For a corner of $E$ which does not intersect the edge $e_{k}=\langle a b\rangle$, we define the sign of the corner to be 0 (see Figure 3 ). We define the sense $\varepsilon_{k}$ of $E$ relative to the edge $e_{k}$ to be the sum of signs of all corners of $E$. This definition is an extension of the notion of sense of a $Q$-disk in [11]. Note that (1) the sign is independent of the orientation of the edge, (2) if two corners of a $Q$-disk on 
opposite edges of the tetrahedron have non-zero sign, then the signs are identical, and (3) if both endpoints of an edge of a $Q$-disk have non-zero sign, then the signs are opposite. Hence the sign determines a slope of a $Q$-disk inside the tetrahedron with respect to an edge.

By an argument similar to that in [11], we can get the system of $Q$-matching equations of $M$ for $\mathfrak{I}$ as follows

$$
\left\{\sum_{i=1}^{3 t} \varepsilon_{k, i} x_{i}=0\right\}_{k}, \quad 0 \leq x_{i}, 1 \leq i \leq 3 t,
$$

where $\varepsilon_{k, i}$ is the sense of a $Q$-disk of $d_{i}^{Q}$-type relative to the edge $e_{k}$. A nonnegative integral solution $\left(x_{1}, x_{2}, \ldots, x_{3 t}\right)$ to the system of $Q$-matching equations is admissible if it satisfies the first constraint that, for each tetrahedron of $\mathfrak{I}$, at most one $x_{i}$ associated with the tetrahedron is non-zero. The $Q$-projective solution space of $M$ is the projectivised solution space, which is a compact, convex, linear cell in $\mathbb{R}^{n}$, of the system of $Q$-matching equations onto the unit polyhedron which is the solution space of $\sum_{i=1}^{3 t} x_{i}=1$. A $Q$-vertex surface in $M$ is a normal surface whose normal $Q$ coordinates is projected onto a $Q$-vertex solution in the $Q$-projective solution space. A finite spanning set of solutions containing the $Q$-vertex solutions can be constructed by elementary methods [8]. We call a solution in the spanning set a $Q$-fundamental solution. These solutions can be obtained from the $Q$-vertex solutions as in the case of regular normal surface theory [4]. We call a normal surface corresponding to a $Q$ fundamental solution a $Q$-fundamental surface. Some interesting surfaces are found in the collection of $Q$-fundamental surfaces.

There is a characterization of $Q$-vertex surfaces and $Q$-fundamental surfaces. A connected two-sided normal surface $F$ is a $Q$-vertex surface (respectively $Q$ fundamental surface) if and only if whenever $X$ and $Y$ are two-sided normal surfaces such that $n F+\Sigma=X+Y$ (respectively $F+\Sigma=X+Y$ ) for some integer $n$ and family of trivial surfaces $\Sigma$, then each component of $X$ and $Y$ is normal isotopic to either $F$ or a component of $\Sigma$.

REMARK 1. The geometric sums of normal surfaces with respect to ideal triangulations are always modulo trivial normal tori. Thus the Euler characteristic formula for geometric sums is valid for the $Q$-theory with respect to ideal triangulations.

We have observed that the normal $Q$-coordinate $\vec{F}_{Q}$ of a normal surface $F$ representing a compact surface is a well-defined finite tuple of non-negative integers which is an admissible solution to the system of $Q$-matching equations. The following theorem gives the converse argument. 
THEOREM 2.1. Let $M$ be an orientable (possibly non-compact) 3-manifold with an ideal triangulation $\mathfrak{I}$ with torus cusps. If $\vec{z}$ is a non-zero admissible solution to the system of $Q$-matching equations then there exists a unique normal surface $F$ (possible non-compact) in $M$ with no trivial components such that $\vec{F}_{Q}=\vec{z}$.

Thus there is a one-to-one correspondence between the set of all normal surfaces and the set of all admissible solutions to the system of $Q$-matching equations for a given non-compact 3-manifold $M$ with an ideal triangulation with torus cusps. It also follows that all $Q$-fundamental surfaces span the set of all normal surfaces in $M$.

\section{The proof of Theorem 2.1}

Let $\vec{z}$ be a non-zero admissible solution to the system of $Q$-matching equations. To construct a normal surface $F$ with $\vec{F}_{Q}=\vec{z}$, we first glue together a collection of $Q$-disks corresponding to $\vec{z}$ along corners and then fill out the holes obtained from the gluing, by attaching $T$-disk components along the curves made up of unglued edges of the $Q$-disks.

We first describe the $Q$-corner gluing rule which is a gluing method of $Q$-disks along corners.

Let $M^{\prime}$ be a compact 3-manifold obtained from $M$ by removing a collection of open regular neighbourhoods of ideal vertices of $\Im$. The boundary of $M^{\prime}$ is a collection of tori each with a pseudo-triangulation induced by $\Im$. Choose a collection $\Omega$ of $Q$-disks in $M^{\prime}$ corresponding to $\vec{z}$. We glue the corners of the $Q$-disks in $\Omega$ together by applying the $Q$-corner gluing rule [11] to the abstract polyhedron $\hat{B}_{k}$ associated with each 1simplex $e_{k}=\langle a b\rangle$. If there is a normal surface $G$ meeting $e_{k}$, then each component of $G \cap \hat{B}_{k}$ is a disk $D_{i}$ which consists of elementary disks glued along the edge $e_{k}$. Since the elementary disks of $D_{i}$ are completely determined by the arcs of $D_{i} \cap \partial \hat{B}_{k}$, $D_{i}$ is fully determined by the simple closed curve $D_{i} \cap \partial \hat{B}_{k}$. So we first construct a collection of pairwise disjoint simple closed curves in $\partial \hat{B}_{k}$ which are obtained from edges of $Q$-disks in $\partial \hat{B}_{k}$ by adding $a$-type arcs and $b$-type arcs in $\partial \hat{B}_{k}$ or isotoping edges of $Q$-disks in $\partial \hat{B}_{k}$ (see Figure 4 ). Note that the collection of closed curves can be constructed because the matching equation of $e_{k}$ provides as many positive slopes as there are negative slopes in $\partial \hat{B}_{k}$. Since the quotient map $q: \hat{B}_{k} \rightarrow B_{k}$ maps a small neighbourhood of $\hat{e}_{k}$ homeomorphically onto a small neighbourhood of $e_{k}$, the gluing rule associated with the edge $\hat{e}_{k}$ in $\hat{B}_{k}$ dictates how the corners on $e_{k}$ in $B_{k}$ are glued together. Two $Q$-disks glued along both boundary points of an edge are glued along this edge. Thus the gluing rule is well-defined globally.

Let $F^{\prime}$ be the union of all $Q$-disks in $\Omega$ after gluing corners of $Q$-disks together. We designate a collection of closed curves from the unglued edges of the $Q$-disks in $F^{\prime}$ to which we will attach embedded trivial components. When we perform the 


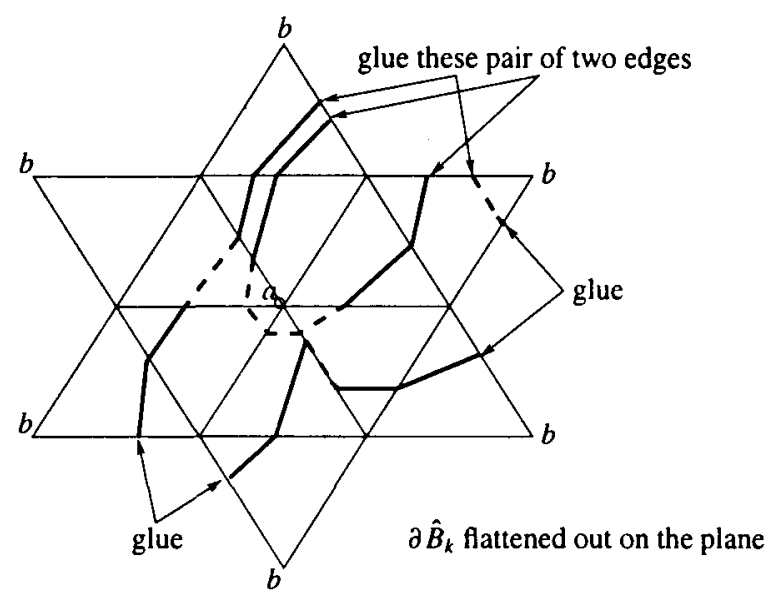

FIGURE 4. $Q$-corner gluing rule along $e_{k}=\langle a b\rangle$

$Q$-corner gluing rule, the construction not only gives the gluing of the comers of $Q$-disks but also shows how the desired normal surface extends around each glued corner of the $Q$-disks. We can complete a disk $D$, possibly with some identification along the boundary, which is the local extension of $F^{\prime}$ around a glued comer $x$ of $Q$-disks by attaching $T$-disks along unglued edges of $Q$-disks around $x$. We can take a pair of edges of $Q$-disks which are glued at the corner $x$ on $e_{k}$ with opposite signs and joined with either $a$-type $T$-disks or $b$-type $T$-disks (see Figure 5 ). Denote such a pair by $\left(\hat{\gamma}_{i}, \hat{\delta}_{i}\right)$ and the image under the quotient map by $\left(\gamma_{i}, \delta_{i}\right)$. Let $\left\{\left(\gamma_{i}, \delta_{i}\right)\right\}$ be the collection of all such unordered pairs. We define an equivalence relation on the collection as follows and several other occurrences; $\left(\gamma_{n}, \delta_{n}\right)$ and $\left(\gamma_{m}, \delta_{m}\right)$ are equivalent if there is a chain $\left\{\left(\gamma_{k_{1}}, \delta_{k_{1}}\right),\left(\gamma_{k_{2}}, \delta_{k_{2}}\right), \ldots,\left(\gamma_{k_{1}}, \delta_{k_{1}}\right)\right\}$ such that $\left(\gamma_{k_{1}}, \delta_{k_{1}}\right)=\left(\gamma_{n}, \delta_{n}\right)$, $\left(\gamma_{k_{i}}, \delta_{k_{i}}\right)=\left(\gamma_{m}, \delta_{m}\right)$ and $\delta_{k_{i}}=\gamma_{k_{i+1}}$, for $i=1,2, \ldots, l-1$. Each equivalence class defined on the collection is a closed chain determining a closed curve made up of edges of $Q$-disks. Let $\Gamma$ be the collection of such closed curves. We attach trivial normal surfaces along each curve of $\Gamma$. To facilitate this and to obtain a trivial surface to attach along a curve of $\Gamma$, we will project the curve onto a torus boundary of $M^{\prime}$.

Let $C$ be a closed curve of $\Gamma$ determined by an equivalence class

$$
\left\{\left(\gamma_{1}, \delta_{1}\right),\left(\gamma_{2}, \delta_{2}\right), \ldots,\left(\gamma_{n}, \delta_{n}\right)\right\}
$$

where $\delta_{i-1}=\gamma_{i}$ for $i=2,3, \ldots, n$ and $\delta_{n}=\gamma_{1}$. If $n=1, \gamma_{1}=\delta_{1}$ itself is a closed curve of $\Gamma$ and normal isotopic to a closed 1-simplex in a torus boundary. We project the curve onto the 1-simplex. Assume $n>1$. For each pair $\left(\gamma_{i}, \delta_{i}\right)$, there is a corresponding pair $\left(\hat{\gamma}_{i}, \hat{\delta}_{i}\right)$ in the abstract polyhedron $\hat{B}_{i}$ associated with the edge $e_{i}=\left\langle a_{i} b_{i}\right\rangle$ such that $x_{i}=\gamma_{i} \cap \delta_{i}$ is on the edge $e_{i}$ and $\hat{x}_{i}=\hat{\gamma}_{i} \cap \hat{\delta}_{i}$ is on the 


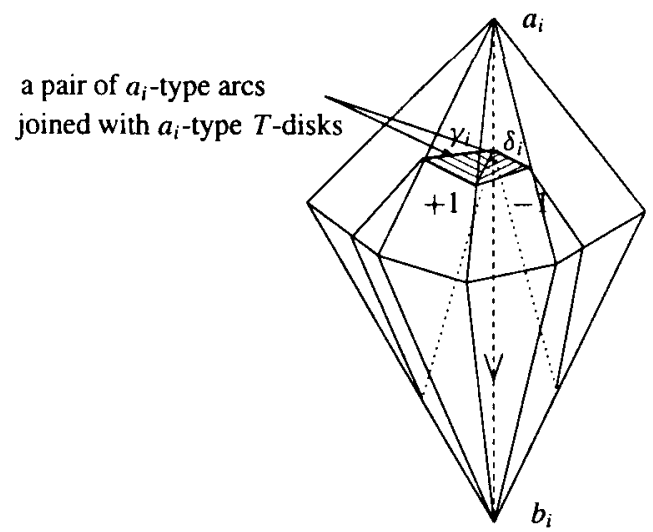

a pair of edges of $Q$-disks

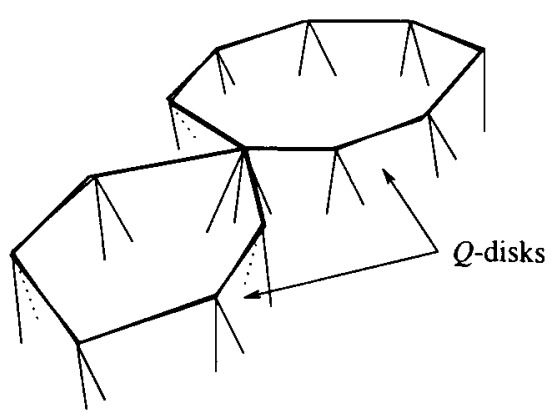

equivalent classes of pairs

FIGURE 5. Classification of edges of $Q$-disks

edge $\hat{e}_{i}$, where $q_{i}\left(\hat{e}_{i}\right)=e_{i}$ and $q_{i}\left(\hat{x}_{i}\right)=x_{i}$ for the quotient map $q_{i}$ defined on $\hat{B}_{i}$. Both $\hat{\gamma}_{i}$ and $\hat{\delta}_{i}$ are either $a_{i}$-type arcs or $b_{i}$-type arcs. There is a natural projection map $p_{i}: \hat{B}_{i} \rightarrow \hat{D}_{a_{i}}$ (respectively $\left.\hat{D}_{b_{i}}\right)$ such that $q_{i} \circ p_{i}\left(\hat{\gamma}_{i}\right)$ and $q_{i} \circ p_{i}\left(\hat{\delta}_{i}\right)$ are arcs on a torus boundary of $M^{\prime}$ and $q_{i} \circ p_{i}\left(\hat{x}_{i}\right)=a_{i}$ (respectively $\left.b_{i}\right)$, where $\hat{\gamma}_{i}$ and $\hat{\delta}_{i}$ are $a_{i}$-type (respectively $b_{i}$-type) arcs, $q_{i}\left(\hat{D}_{a_{i}}\right)=D_{a_{i}}$ (respectively $q_{i}\left(\hat{D}_{b_{i}}\right)=D_{b_{i}}$ ) and $D_{a_{i}} \cup D_{b_{i}}=B_{k} \cap \partial M^{\prime}$. Let $C^{*}$ be the union of images of all such pairs $\left(\hat{\gamma}_{i}, \hat{\delta}_{i}\right)$ under the map $q_{i} \circ p_{i} . C^{*}$ is a well-defined closed curve in a torus boundary $T_{v}$ of $M^{\prime}$. We attach a trivial component along $C$ according to the following three cases.

Case 1. $C^{*}$ is a trivial simple closed curve in $T_{v}$.

In this case, $C^{*}$ separates $T_{v}$ into two components, a disk $D$ and a torus with a hole $T_{0}$. Let $\hat{E}_{\gamma_{i}}$ and $\hat{E}_{\delta_{i}}$ be the $Q$-disks in $\hat{B}_{i}$ along $\hat{\gamma}_{i}$ and $\hat{\delta}_{i}$, whose images under the quotient map $q_{i}$ are the $Q$-disks of $F^{\prime}$ along $\gamma_{i}$ and $\delta_{i}$ respectively. Then the image of $\hat{E}_{\gamma_{i}}$ under the map $q_{i} \circ p_{i}$ must be on the same side of $C^{*}$ as the image of $\hat{E}_{\delta_{i}}$. This is true for all $i$. Thus the union of images of $Q$-disks along $\left(\hat{\gamma}_{i}, \hat{\delta}_{i}\right)$ is in either $D$ or $T_{0}$. We attach either $D$ or $T_{0}$, which is on the opposite side, along $C$ by isotoping its boundary along 2-simplices containing the arcs of the equivalence class of $\left(\gamma_{i}, \delta_{i}\right)$.

Case 2. $C^{*}$ is an essential simple closed curve in $T_{v}$.

In this case, we will attach a half infinite normal annulus along $C$ as follows.

Take an infinite sequence of pairwise disjoint tori $\left\{T_{v} \times 1 / n \mid n \in \mathbf{Z}_{+}\right\}$in the regular neighbourhood of the ideal vertex $v$ bounded by $T_{v}$ such that $T_{v} \times 1=T_{v}$ and $\lim _{n \rightarrow \infty} T_{v} \times 1 / n=v$. We can define a projection map from each torus $T_{v} \times 1 / n$ onto $T_{\nu}$ in the obvious way. There is a simple closed curve $C_{n}$ in $T_{v} \times 1 / n$, for $n=2,3, \ldots$, whose image by the projection map is the simple closed curve $C^{*}$ in $T_{v}$. 


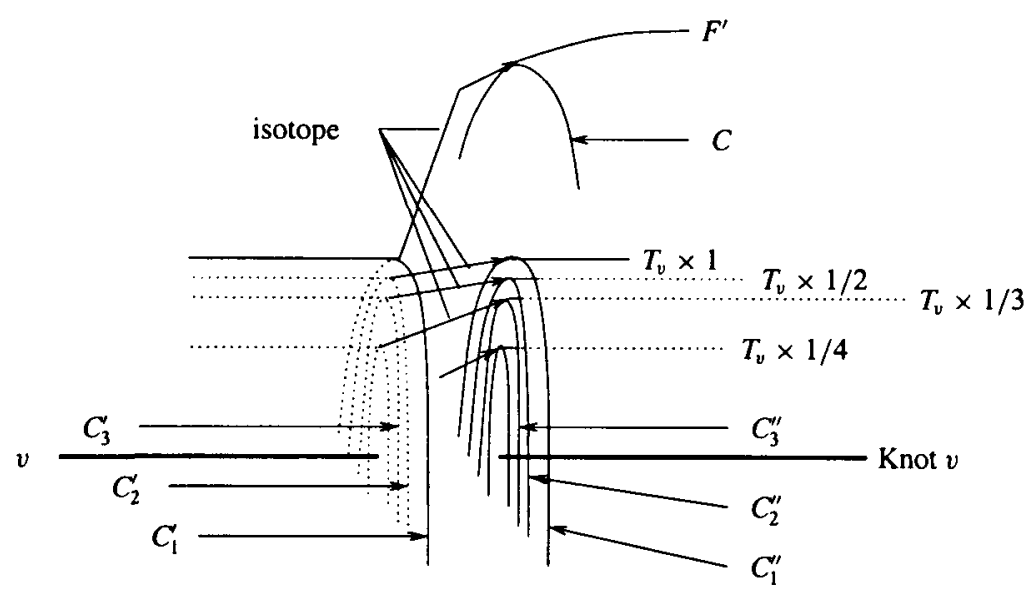

FIGURE 6. Construction of an infinite normal annulus

Let $C_{1}=C^{*}$ in $T_{v} \times 1=T_{v}$. Cut each torus $T_{v} \times 1 / n$ along the curve $C_{n}$ to obtain two copies of $C_{n}$, say $C_{n}^{\prime}$ and $C_{n}^{\prime \prime}$ (see Figure 6). To construct a half infinite normal annulus, we join these curves in one of the following ways.

The first way is to join $C_{n}^{\prime}$ and $C_{n-1}^{\prime \prime}$ for all $n>1$. We isotope each normal arc of $C_{n}^{\prime}$ to the parallel normal arc of $C_{n-1}^{\prime \prime}$ along the 2-simplex of $\Im$ containing these two arcs. The isotopy preserves the elementary disk types of the disks isotoped along the curves and does not introduce any intersection of $Q$-disks in $F^{\prime}$. This process produces the desired infinite trivial normal annulus $A_{1}$ with the boundary $C_{1}^{\prime \prime}$ which is disjoint from $F^{\prime}$. We can get the second infinite trivial normal annulus $A_{2}$ with the boundary $C_{1}^{\prime}$, by joining $C_{n}^{\prime \prime}$ to $C_{n-1}^{\prime}$ for all $n>1$ in the same manner.

We attach either $A_{1}$ or $A_{2}$ along $C$ depending on the images of the $Q$-disks along $C$ under the maps $q_{i} \circ p_{i}$ using the same technique as used in Case 1. Note that the slope of the resulting normal surface is the slope of $C^{*}$.

Case 3. $C^{*}$ is not a simple closed curve in $T_{v}$.

In this case, we can modify $C^{*}$ to a simple closed curve by attaching subtriangles along arcs of $C$. (For a detailed description, see [9].) Then we follow the procedure in either Case 1 or Case 2 to attach a trivial component along $C$.

Repeat this construction for all curves of $\Gamma$. We may have intersections among the trivial surfaces, disks or infinite annuli, which we have attached along each curve of $\Gamma$. We may assume that they intersect transversely, that is, the elementary disks along the intersection curves intersect transversely in abstract tetrahedra. We can perform a regular exchange along all such intersection curves. Discard any trivial components resulting from the regular exchange. The resulting surface is the desired normal surface $F$ embedded in $M$. 
The uniqueness of $F$ follows from the construction; the $Q$-corner gluing rule, the way of choosing the collection $\Gamma$ and the way of attaching trivial components along the curves in $\Gamma$.

REMARK 2. We obtained an algorithm, from the above proof, to construct a normal surface corresponding to a given admissible solution to the system of $Q$-matching equations of an orientable 3-manifold with an ideal triangulation with torus cusps. Note that these normal surfaces can be completely specified by considering only the $Q$-disks glued at corners without attaching any triangular disks. A finite set of normal surfaces containing all the $Q$-fundamental surfaces can be constructed. One only needs to take the set of all normal surfaces whose $Q$-weight is less than or equal to the sum of the $Q$-weights of all the $Q$-vertex surfaces. (This was observed by Jaco in [4].) We can obtain the remaining normal surfaces by geometric sums of the $Q$-fundamental surfaces.

\section{Some extensions of the $Q$-theory}

A semi-ideal-triangulation is a pseudo-triangulation allowing both ideal vertices and real vertices. We can extend the normal surface $Q$-theory developed for ideal triangulations to this cell structure. The only additional feature is the construction of the desired normal surface around real vertices. The idea for the construction of a trivial surface to attach along a closed curve made up of edges of $Q$-disks and projected onto a 2 -sphere neighbourhood of a real vertex is exactly the same as the case of an ideal vertex in the proof of Theorem 2.1 but all the trivial surfaces are disks.

THEOREM 4.1. Let $M$ be an orientable 3-manifold with a semi-ideal-triangulation with torus cusps. If $\vec{z}$ is a non-zero admissible solution to the system of $Q$-matching equations then there exists a unique normal surface $F$ in $M$ with no trivial components such that $\vec{F}_{Q}=\vec{z}$.

We now extend the $Q$-theory to almost normal surfaces. A properly embedded surface $F$ in a 3-manifold with an ideal triangulation is an almost normal surface if $F$ intersects each tetrahedron in a collection of elementary disks of $T$-disk types and $Q$-disk types and, in one of the tetrahedra, precisely one disk with boundary curve consisting of eight normal arcs, which is called a octagonal disk ( $O$-disk) and possibly some $T$-disks. We add three more elementary disk types of $O$-disk to obtain this more general notion of surfaces (see Figure 7). This definition follows [10].

An octagonal disk $E$ has two corners in each of two opposite 1-simplices such that the both normal arcs joined at each corner are of the same type relative to the edge where the corner is. In this case, we define the sign of the corner to be 0 . For the 

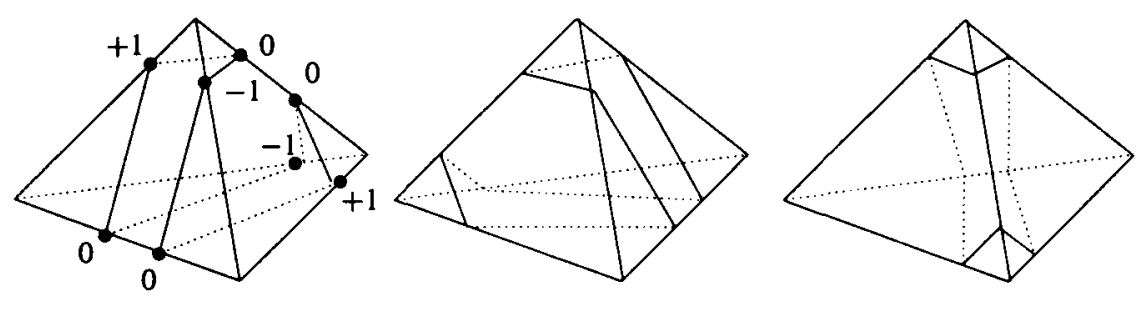

FIGURE 7. 8-sided disk types

other four corners of $E$, we apply the same method as the case of a $Q$-disk to define the signs of corners (see Figure 7). We define the sense $\varepsilon_{k}$ of $E$ relative to $e_{k}$ to be the sum of all the signs of the corners of $E$ which meet the edge $e_{k}$. Let $d_{1}, d_{2}, \ldots, d_{3 t}$ and $d_{3 t+1}, d_{3 t+2}, \ldots, d_{6 t}$ be a fixed order of quadrilateral disk types and octagonal disk types, respectively, of a 3-manifold $M$ with an ideal triangulation having $t$ tetrahedra. As in the case of normal surfaces we define normal $Q$-coordinates of $6 t$-tuples for almost normal surfaces whose positive entries represent at most one quadrilateral or octagonal disk type from each tetrahedron. The following is the system of $Q$-matching equations for almost normal surfaces of $M$ :

$$
\left\{\sum_{i=1}^{6 t} \varepsilon_{k, i} x_{i}=0\right\}_{k}, \quad 0 \leq x_{i}, 1 \leq i \leq 6 t,
$$

where $\varepsilon_{k, i}$ is the sense of a disk of type $d_{i}$ relative to a 1-simplex $e_{k}$. An admissible solution to the system is a non-negative integral solution $\left(x_{1}, x_{2}, \ldots x_{6 t}\right)$ satisfying the condition, that for each tetrahedron $\Im$, at most one $x_{i}$ associated with the tetrahedron is non-zero, and for exactly one tetrahedron, exactly one of the three components associated with $O$-disk types in the tetrahedron is non-zero. We can construct an almost normal surface embedded in $M$ corresponding to a given admissible solution to the system of $Q$-matching equations. The idea of the construction is similar to the proof of Theorem 2.1 .

THEOREM 4.2. Let $M$ be a non-compact orientable 3-manifold with an ideal triangulation $\mathfrak{I}$ with torus cusps. If $\vec{z}$ is a non-zero admissible solution to the extended system of $Q$-matching equations for almost normal surfaces in $M$ then there exists a unique almost normal surface $F$ (possibly non-compact) in $M$ with no trivial components such that $\vec{F}_{Q}=\vec{z}$.

PROOF. The only additional case from the proof of Theorem 2.1 is the gluing of corners on the edges meeting octagonal disks.

Let $O$ be the octagonal disk in a collection $\Omega$ of elementary disks corresponding to the solution $\vec{z}$. When we glue the corners of $Q$-disks on an edge $e_{k}$, the gluing pattern 
is described in terms of matching edges of the $Q$-disks around $e_{k}$ and does not depend on the whole structure of the disks but only on the arc types of edges of the $Q$-disks. This makes it possible to extend the gluing rule to the case of almost normal surfaces.

Let $\hat{B}_{k}$ be the abstract polyhedron associated with the edge $e_{k}$ and $\hat{e}_{k}$ the center edge of $\hat{B}_{k}$. Two spanning edges intersecting at a corner on $\hat{e}_{k}$ determine an elementary disk type of either $T$-disk or $Q$-disk. For a corner $x$ of the octagonal disk $O$ on $\hat{e}_{k}=\langle a b\rangle$, we replace the disk $O$ with an elementary disk of either $T$-disk type or $Q$-disk type, which conforms with the arcs of $O$ cornered at $x$. If $x$ is of sign 0 , we replace $O$ with a $T$-disk and otherwise, we replace $O$ with a $Q$-disk which has the same sign at the corner. We now perform the gluing rule applied to the case of normal surfaces. When we construct a collection of simple closed curves in $\partial \hat{B}_{k}$, in a stack of zero slopes, the one coming from an $O$-disk has to be closest to the equator.

REMARK 3. The theorem also holds for a semi-ideal-triangulation with torus cusps of a 3-manifold.

REMARK 4. If we allow an almost normal surface to contain a single exceptional piece which is a pair of normal disks with an unknotted tube as well as an octagon, the $Q$-theory applies without any constraints. Since both the $Q$-corner gluing and the attaching of $T$-disks depends only on the arc types, we can replace, as we glue the corners of disks, each tube by normal disks corresponding to the boundary normal curves of the tube.

\section{Some results of the $Q$-theory}

A knot complement in $S^{3}$ is always given an ideal triangulation with a torus cusp by the SnapPea software package [12]. We apply the normal surface $Q$-theory for ideal triangulations to knot complements.

In a compact, irreducible, $\partial$-irreducible, triangulated 3-manifold, any incompressible, $\partial$-incompressible, compact surface can be moved by an isotopy to a normal surface [6]. But in a knot complement with an ideal triangulation, the existence of a normal surface representing a minimal Seifert surface of the knot is not guaranteed. The figure-eight knot is a counterexample which does not contain any normal or almost normal surface representing a minimal Seifert surface of the knot [8].

Many applications of normal surface theory result from interesting surfaces occurring in the collection of fundamental surfaces. This leads to some efficient algorithms deciding topological properties of given 3-manifolds [7].

In this section we prove the existence of a $Q$-fundamental surface representing a non-trivial essential torus in a knot complement of a non-simple knot. This results in an algorithm to decide if a given knot is a simple knot. 
The following result is due to Burton and Rubinstein [2].

THEOREM 5.1. Given an ideal triangulation $\mathfrak{\Im}$ of a non-compact orientable irreducible and $\partial$-irreducible 3-manifold $M$, we can construct an ideal triangulation with no embedded non-trivial normal or almost normal 2-spheres in $M$.

Moreover, Casson observed the following result which is proven in [1] using an interesting argument.

THEOREM 5.2. For a non-compact 3-manifold admitting a complete hyperbolic metric of finite volume, given an ideal hyperbolic triangulation, there are no immersed or embedded normal or almost normal 2-spheres or tori, except for peripheral ones which consist entirely of triangular normal disks.

Let $F$ be a normal surface in a 3-manifold $M$ and $\Omega$ be the union of all $Q$-disks in the induced cell structure of $F$. We call a connected component of $\Omega$ a $Q$-disk component of $F$.

PROPOSITION 5.3. Let $M$ be a 3-manifold with an ideal triangulation (semi-idealtriangulation) with torus cusps and let $F$ be a $Q$-fundamental surface in $M$. Then $F$ has only one $Q$-disk component, that is, the union of all $Q$-disks of $F$ is connected.

PROOF. For each $Q$-disk component of $F$, we can construct a unique normal surface represented by the finite-tuple corresponding to the component. If $F$ has more than one $Q$-disk component then there is a non-trivial geometric sum of normal surfaces which results in $F$ modulo trivial normal surfaces.

For the use in the next theorem, we recall that $F+\Sigma=X+Y$ is in reduced form, where $\Sigma$ is a (possibly infinite) collection of trivial normal tori, if $X$ intersects with $Y$ in a minimal set of intersection curves, that is, we cannot remove any intersection curves in $X \cap Y$ by isotopies.

THEOREM 5.4. Let $M$ be a non-compact irreducible and $\partial$-irreducible 3-manifold with an ideal triangulation $\Im$ with torus cusps. Suppose that $F$ is a closed normal surface having the least weight or the least $Q$-weight among all normal surfaces isotopic to $F$ and $F+\Sigma=X+Y$ is in reduced form, where $\Sigma$ is a (possibly infinite) collection of trivial normal tori. If $F$ is two-sided and incompressible, then both $X$ and $Y$ are incompressible.

PROOF. Note that normal surfaces $X$ and $Y$ intersect in open intervals or in simple closed curves. If $X \cap Y$ contains an open interval, then there is a cusp of $M$ on which $X+Y=F+\Sigma$ has boundary. However, all components of $F+\Sigma$ are closed, and 
hence $X \cap Y$ only contains simple closed curves. The proof is now largely based on the proofs of Lemma 2.1 and Theorem 2.2 in [5], which the reader may wish to refer to.

Assume that there is a compressing disk for $X$. Among all compressing disks, we choose a disk $D$ such that $D$ is transverse to $Y$, and such that it has the least number of components of intersection with $Y$.

Using the fact that $F$ is incompressible, we have the two following cases. In the first case, we have $D \cap Y=\emptyset$, and $\partial D$ is a boundary slope of $X$. But this implies that $D$ is a compressing disk for a component of $\partial M$, which is not possible since $M$ is $\partial$-irreducible.

Hence, we are in the second case, where $D \cap Y$ is not empty. Note that $D \cap Y$ cannot contain any simple closed curves, and hence consists of finitely many spanning arcs for $D$. Let $p$ be an endpoint of a spanning arc of $D \cap Y$. Then $p$ is in $X \cap Y$ and a regular exchange along the curve of $X \cap Y$ containing $p$ puts one of the two regions of $D-(D \cap Y)$ associated to $p$ into a region, say bad region associated to $p$, joined to the outside of $D$ around $p$. Following the argument of Jaco-Oertel [5], there is a component $E$ of $D-(D \cap Y)$ containing at most one bad region.
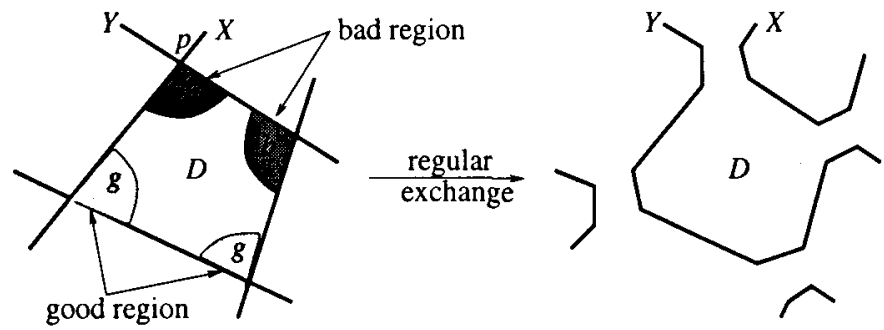

FIGURE 8. Bad region associated to $p$

Assuming $E$ has no bad region, then there is a disk $\Delta$ in $M$ with boundary either on a component of $F$ or on a boundary parallel torus $T \subset \Sigma$. Hence, $\partial \Delta$ bounds a disk on either $F$ or $T$ because both $F$ and $T$ are incompressible. As in [5] (using the fact that the geometric sum is reduced and the manifold is irreducible), we get a contradiction to the choice of $D$.

Thus, there is a region $E$ with exactly one bad region at a point $p$ on a curve $C$ in $X \cap Y$. As in [5], this region yields a disk $\Delta$, whose boundary consists of an $\operatorname{arc} \delta^{\prime}$ on some annulus $A^{\prime}$ in $M$ and an $\operatorname{arc} \delta$, which is either on a component of the closed surface $F$ or on some boundary parallel torus $T \subset \Sigma$. If $\delta$ is contained on $T$, the minimality of $D$ implies that $T$ is at an end of $M$ where both $X$ and $Y$ have a non-trivial boundary slope, and that $C$ cuts an infinite normal annulus off by portions of $\partial D$ and $C$. We can perform an isotopy of $\partial D$ across $E$ which determines an isotopy of $D$ and reduces the number of arcs in $D \cap Y$. But this contradicts our choice of $D$. 
Hence, $\delta$ is contained on $F$. However, in case of $F$ is of least weight, the argument of [5] applies now (using the fact that $M$ is irreducible and that $F$ is closed) to give a contradiction to the fact that $F$ was of minimal weight. Now assume that $F$ is of least $Q$-weight. We have already introduced the annulus $A^{\prime}$ in $M$ with zero weight. We can observe that the weight-reducing isotopy in [5] across the meridian disk $\Delta$ of a solid torus $\hat{T}$ with the boundary $\partial \hat{T}=A \cup A^{\prime}$ for some annulus $A$ in $F$ also reduces the $Q$-weight which also gives a contradiction. Let $F^{\prime}$ be the surface (which is not normal) obtained by an isotopy moving $A$ to $A^{\prime}$. We claim that a normal surface $F^{\prime \prime}$ obtained from $F^{\prime}$ by normalization process is of less $Q$-weight than $F$. Otherwise, the annulus $A$ is involved with only triangular disks along one cusp and then follow the same argument as the previous case that $\delta$ is on a boundary parallel torus $T$.

As we arrive at a contradiction in each case, it follows that $X$ must be incompressible. By the symmetry of the situation, the same applies to $Y$.

By Theorem 5.1, we can always construct an ideal triangulation allowing no embedded non-trivial normal 2-spheres for a given arbitrary ideal triangulation of a non-trivial knot complement in $S^{3}$. Hence the following theorem gives an algorithm to decide if a given knot is a simple knot.

THEOREM 5.5. Let $M$ be a knot complement of a non-simple knot $K$ in $S^{3}$ which has an ideal triangulation $\Im$ allowing no normal 2-spheres. Then there is a $Q$-fundamental surface representing a non-trivial essential torus.

ProOF. We can normalize any closed incompressible surface in $M$ by the same processes as in the case of compact triangulated 3-manifolds. Since the knot is nonsimple, $M$ contains a non-trivial essential torus. Let $F$ be a normal surface having the least $Q$-weight among all normal surfaces representing non-trivial essential tori. Suppose that $F+\Sigma=X+Y$ in reduced form, where $\Sigma$ is a collection of trivial tori and $X$ and $Y$ are non-trivial normal surfaces. Since there are no normal 2-spheres and no normal disks embedded in $M$, the Euler characteristic of any component of $X$ and $Y$ is negative unless it is a torus and $0=\chi(F)+\chi(\Sigma)=\chi(X)+\chi(Y)$. Thus each component of both $X$ and $Y$ is a non-trivial torus which is essential by the previous theorem, and has $Q$-weight less than $F$ which contradicts the fact that $F$ has the least $Q$-weight.

REMARK 5. Algorithm to decide if a given knot $K$ is a simple knot (we may assume that the knot is not trivial due to an algorithm deciding if a given knot is trivial):

Step 1. Find an ideal triangulation $\Im$ of the knot complement $M=S^{3}-K$ with no normal 2-spheres. We can obtain such a triangulation from an arbitrary ideal triangulation by collapsing all possible normal 2 -spheres by the process given in [2]. 
Step 2. Construct all $Q$-fundamental surfaces in $M$ with respect to $\Im$ using the process given in the Section 3.

Step 3. If there is a non-trivial essential torus among the $Q$-fundamental surfaces constructed in the Step 2, $K$ is a non-simple knot. Otherwise, $K$ is simple.

\section{Acknowledgements}

The author would like to thank Jeffrey Tollefson and Hyam Rubinstein for their guidance and discussions. She also wants to thank the referee for helpful suggestions, especially in Theorem 5.4.

\section{References}

[1] B. Burton, W. Jaco, D. Letscher and J. H. Rubinstein, 'Algorithms to find connected sum and torus decompositions of 3-manifolds', preprint, 2000.

[2] B. Burton and J. H. Rubinstein, in preparation.

[3] W. Haken, 'Theorie der Normalflächen', Acta Math. 105 (1961), 245-375.

[4] W. Jaco, 'Personal notes', unpublished, 1987.

[5] W. Jaco and U. Oertel, 'An algorithm to decide if a 3-manifold is a Haken manifold', Topology 23 (1984), 195-209.

[6] W. Jaco and J. H. Rubinstein, 'PL equivariant surgery and invariant decompositions of 3-manifolds', Advances in Math. 73 (1989), 149-191.

[7] W. Jaco and J. L. Tollefson, 'Algorithms for the complete decomposition of a closed 3-manifold', Illinois J. Math. 39 (1995), 358-406.

[8] E. Kang, 'Normal surfaces in the figure-8 knot complement', J. Knot Theory Ramifications 12 (2004), 269-279.

[9] - Normal surfaces in knot complements (Ph.D. Thesis, University of Connecticut, 1999).

[10] A. Thompson, 'Thin position and the recognition problem for $S^{3}$, Math. Res. Lett. 1 (1994), 613-630.

[11] J. L. Tollefson, 'Normal surface $Q$-theory', Pacific J. Math. 183 (1998), 359-374.

[12] J. R. Weeks, 'Snappea: A computer program for creating and studying hyperbolic 3-manifolds', available by anonymous ftp from geom. umn. edu/pub/sof tware/snappea.

Department of Mathematics

College of Natural Sciences

Chosun University

Gwangju 501-759

Korea

e-mail: ekang@chosun.ac.kr 